a frank attempt to rehabilitate the concept of race as a primary descriptive category explaining and integrating differences in everything from IQ to the angular elevation of the erect penis. It proceeds by skimming the surface of countless studies of grab groups, and what is to be found on that surface is not always cream.

The broad drift of Rushton's argument is that the Caucasoid and Mongoloid races of man have evolved against harsher environmental pressure than the Negroid, leading to a typical ordering of racial characteristics related to the relative emphasis on $r$ (gamete production, mating behaviour and high reproductive rates) or $K$ (parental care, resource acquisition, kin provisioning and social complexity) reproductive strategies. Guess at which end the blacks come?

The supposed relationship between cranial capacity and IQ gets quite an airing. First Rushton sets out to show that Caucasoids and Mongoloids have bigger heads than Negroids. Next he claims that the evidence shows that Caucasoids and Mongoloids do better on standard psychometric tests than Negroids. Then one is invited to the conclusion that blacks are less intelligent because they have smaller heads. The fact that women have smaller heads than men is brushed aside: perhaps we should correct for body size; or perhaps the excess neurons men have are related to the spatial and mathematical thinking at which men excel.

The temptation is to undertake a demolition job on Rushton's approach to the analysis of statistical data: there is plenty of scope, and it would be great fun, but I am sure there are plenty of others who can do it just as well as I. Much more insidious is the uncritical, not to say unthinking, credence Rushton and others lend to inferences from standard test scores. According to some of the data he presents, around half the population of Africa are sufficiently mentally subnormal to need supervision or care. It is the same mistake that Herrnstein and Murray make in The Bell Curve, attributing measurement properties to tests beyond what has been or could be shown, and not recognizing the extent to which their content including so-called culture-fair or culture-free tests - reflects our built intellectual environment.

Race, Evolution, and Behavior does a major disservice to the serious study of the biological basis of behaviour and left me shuddering. It is time sociobiologists took a long hard look at the standards they adopt in evaluating psychometric evidence that suits their enterprise. And I should know: I take a size 8 in hats.

Steve Blinkhorn is at Psychometric Research and Development Ltd, Brewmaster House, The Maltings, St Albans, Hertfordshire AL1 $3 H T$, UK.

\section{Buried treasures}

\section{Keith Stewart Thomson} Documenting Evolutionary Patterns. By Andrew W. Smith. Blackwell Scientific: 1994. Pp. 223. £19.95 (pbk).

IT is now almost 30 years since Hennig's cladistic methods of systematic analysis burst on the scene, bringing both turmoil and order to a field, and replacing gestalt with logic. Cladistics is based on a deceptively simple proposition - that in looking for order in biological (or other) diversity one should group together only those species that share uniquely derived features. 'Similarity' (the old way of looking at things) must therefore be rooted in true phylogenetic connection, not convergence or the sharing of retained primitive characters. The rigour of analysis forces both the quantity and quality of data. It requires comparability of datasets.

As a result of this approach, order and pattern are now emerging from even the most intractable groups of organisms. (It is not by chance that Hennig was an entomologist.) But an odd result has developed. Simply speaking, there have been two main reasons for engaging in systematic studies of any group. The first is to find the pattern of relationships among
Systematics and the Fossil Record:

the species - "A is more closely related to B than to C". The second is to find evolutionary relationships, which fundamentally means the biblical "A begat B, who begat C". Both sorts of information have been thought, at various times, to be essential to analysis of a whole range of subjects - biogeographical relationships, for example. Cladistics, however, is capable of analysing only the first of these two. It determines 'sister-group' relationships but is logically incapable of determining 'ancestor-descendant' relationships. In the branching diagrams that form the stock-in-trade of cladistics, none of the nodes is named. So, ironically, what is called phylogenetic systematics produces not what we used to call 'phylogenies' but, rather, metaphylogenies ('patterns', for convenience).

Historically, evolutionary analysis and particularly palaeontology have been a mixture of analysis and narrative: logic and story-telling. Partly under the influence of cladistics and the quality of data it produces, palaeontology has moved away from the narrative mode. But two controversial problems remain: how can one in practice combine the sorts of data that palaeontology produces with those from recent organisms? And, in principle, is palaeontology even necessary to the nowmisnamed phylogenetic systematics, or simply a distraction at best?

Smith's book is not only a superb

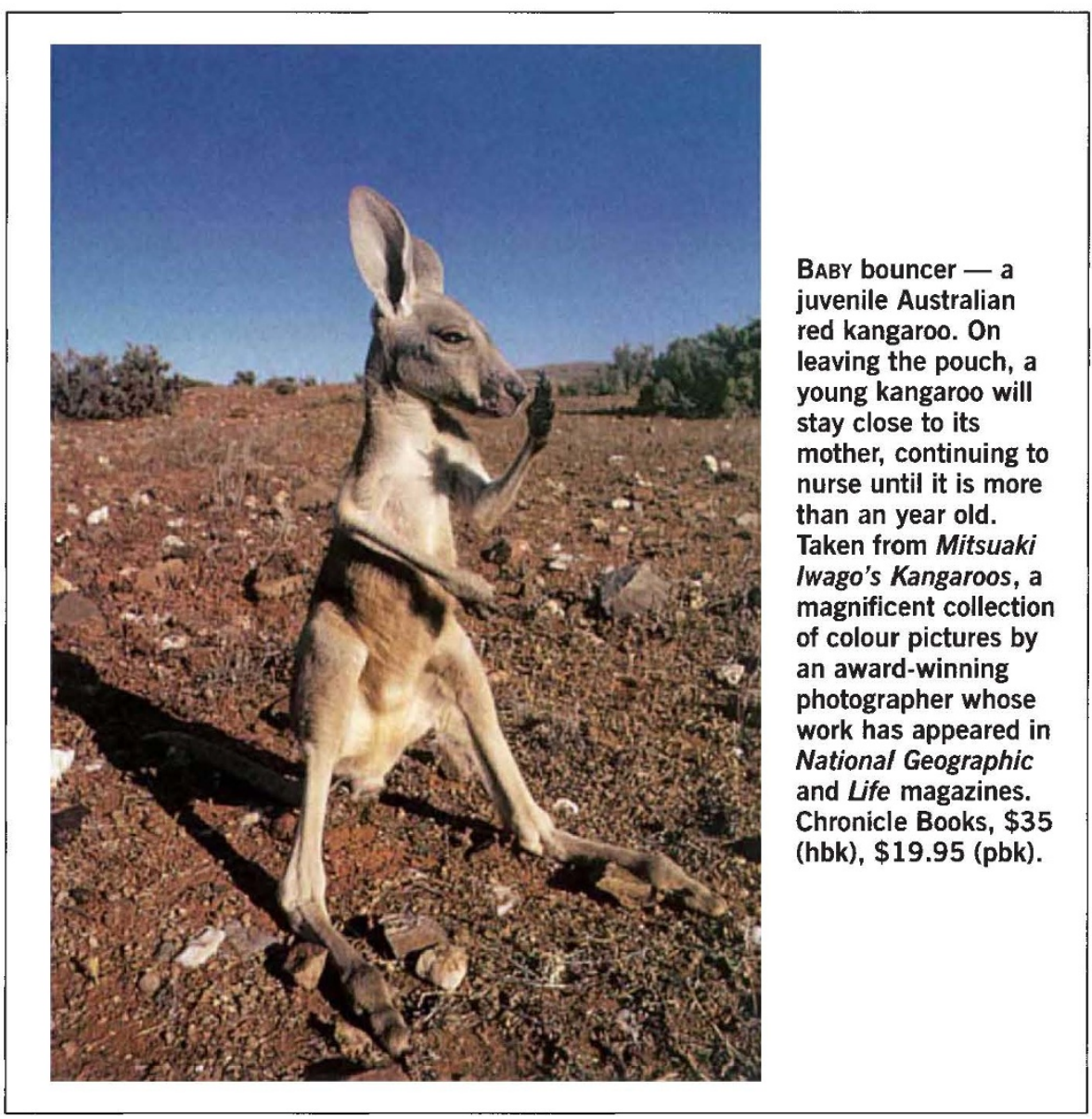

